\title{
NeuroRegulation
}

\section{Proceedings of the 2016 ISNR Conference: Poster Presentations}

\author{
Selected Abstracts of Conference Presentations at the 2016 International Society for Neurofeedback \\ and Research (ISNR) 24th Conference, Orlando, Florida, USA
}

Citation: International Society for Neurofeedback and Research. (2016). Proceedings of the 2016 ISNR Conference: Poster Presentations. NeuroRegulation, 3(4), 186-194. http://dx.doi.org/10.15540/nr.3.4.186

Copyright: $\odot$ 2016. ISNR. This is an Open Access article distributed under the terms of the Creative Commons Attribution License (CC-BY).

\section{Auditory Integration Training (AIT) in Children with Autism Spectrum Disorder: Effects on Auditory Evoked Potentials and Mismatch Negativity \\ Estate Sokhadze ${ }^{1}$, Manuel Casanova ${ }^{1}$, Allan Tasman ${ }^{2}$, and Sally Brockett ${ }^{3}$ \\ ${ }^{1}$ University of South Carolina School of Medicine, Greenville, South Carolina, USA \\ ${ }^{2}$ University of Louisville, Louisville, Kentucky, USA \\ ${ }^{3}$ IDEA Training Center, North Haven, Connecticut, USA}

Background. Autism is a pervasive developmental disorder of childhood characterized by deficits in social interaction, language, and stereotyped behaviors along with a restricted range of interests. It is further marked by an inability to perceive and respond to social and emotional signals in a typical manner. This might due to the deficits of sensory information integration. According to several recent theories, sensory processing and integration abnormalities may play an important role in impairments of perception, cognition, and behavior in individuals with autism spectrum disorder (ASD). Among these sensory abnormalities auditory perception distortion may contribute to many typical symptoms of Autism. The pilot study used Berard's technique of auditory integration training (AIT, Brockett, Lawton-Shirley, \& Giencke-Kimball, 2014) to improve sound integration in children with Autism. It also aimed to understand the abnormal neural and functional mechanisms underlying sound processing distortion in Autism by incorporating behavioral, psychophysiological, and neurophysiological outcomes.

Methods. It was proposed that exposure to twenty 30-min AIT sessions would result in improved behavioral evaluation scores and positively affect both early ( $\mathrm{N} 1$, mismatch negativity [MMN, Näätänen, Paavilainen, Rinne, \& Alho. 2007]) and late (P3) components of evoked potentials in auditory oddball task. Twenty children with ASD participated in the AIT research study. A group of 16 typically developing children served as a contrast group in the auditory oddball task. Eighteen participants with ASD completed 20 sessions of the training and allowed collection of all required outcomes.

Results. Behavioral questionnaires showed significant symptom severity decrease post-AIT. Comparison of evoked potential characteristics of children with ASD vs. typically developing children revealed several interesting group difference findings, more specifically a delayed latency of $\mathrm{N} 1$ to rare and frequent stimuli, larger mismatch negativity; higher P3a to frequent stimuli, and at the same time delayed latency of $\mathrm{P} 3 \mathrm{~b}$ to rare stimuli in the Autism group. Post-AIT changes in evoked potentials could be summarized as a decreased magnitude of $\mathrm{N} 1$ to rare stimuli, marginally lower negativity of $\mathrm{MMN}$, and decrease of the P3a to frequent stimuli along with shorter latency of the P3b to the rare stimuli. These evoked potential changes following completion of Berard AIT course are in a positive direction, making them less distinct from those recorded in agematched group of typical children, thus could be considered as changes towards normalization. Parental questionnaires clearly demonstrated improvements in behavioral symptoms such as irritability, hyperactivity, repetitive behaviors, and other important behavioral domains.

Conclusions. The results of the study propose that more controlled research is necessary to document behavioral and psychophysiological changes resulting from AIT and to provide explanation of the neural mechanisms of how sensory integration training may affect behavior and psychophysiological responses of children with ASD. 


\section{References}

Brockett, S. S., Lawton-Shirley, N. K., \& Giencke-Kimball, J. (2014). Berard Auditory Integration Training: Behavior changes related to sensory modulation. Autism Insights, 6, 110. http://dx.doi.org/10.4137/AUI.S13574

Näätänen, R., Paavilainen, P., Rinne, T., \& Alho, K. (2007). The mismatch negativity (MMN) in basic research of central auditory processing: A review. Clinical Neurophysiology, 118(12), 2544-2590. http://dx.doi.org/10.1016 /j.clinph.2007.04.026

\section{Event-Related Potential and Induced Gamma Oscillations During Emotional Facial Expression Processing in Autism Spectrum Disorder \\ Estate Sokhadze ${ }^{1}$, Manuel Casanova ${ }^{1}$, Ayman El-Baz ${ }^{2}$, and Yao Wang ${ }^{2}$ \\ ${ }^{1}$ University of South Carolina School of Medicine, Greenville, South Carolina, USA \\ ${ }^{2}$ University of Louisville, Louisville, Kentucky, USA}

Background. Autism spectrum disorder (ASD) is one neurodevelopmental disorder which presents with impairments in communication and social skills as well as stereotyped, repetitive patterns of behavior. Disturbances of affective reactivity and innate inability to perceive and respond to social cues including facial emotional expressions in a typical and appropriate manner are the hallmark deficits of ASD. There are several theories describing the neurobiology of underlying deficits. The study used event-related potentials (ERP) and single-trial induced EEG gamma oscillations recording in a modification of a "Theory-of-mind" (ToM) test (Sabbagh, 2004) using facial emotional expression recognition to test emotional responsiveness in children with Autism and typical age-matched children.

Methods. Autism is featured by difficulty in decoding affective facial cues. The goal of the study was to find the differences between ASD group ( $N=$ 19 , mean age $=16.3$ years, $S D=4.9$ years) and typically developing children (CNT group, $N=21$, mean age $=14.9$ years, $S D=4.5$ years) in behavioral (reaction time and accuracy), induced gamma and ERP correlates of processing emotional information from facial expressions. Children with $\operatorname{ADHD}(N=14$, mean age $=14.4$ years, $S D=3.9$ years) served as a contrast group in induced gamma analysis. Task had four different conditions: either to identify the gender or the emotion of the face. Dense-array EEG was recorded using EGI system. The ERP components analyzed in the study were parieto-occipital N170, frontal P3a, and parietal P3b, while induced gamma oscillations were recorded at eight frontal and parietal sites.

Results. ERP measures yielded the following group differences: N170 showed a more negative amplitude in the ASD group than controls when identifying emotional faces $(F=5.66, p=.023)$. The latency of N170 was prolonged in the ASD group ( $F$ $=7.54, p=.01$ ). The ASD group had a larger frontal P3a amplitude as compared to controls when differentiating emotions $(F=5.15, p=.03)$. In the emotion recognition conditions, $\mathrm{P} 3 \mathrm{~b}$ had larger amplitude in Autism $(F=4.17, p=.049)$. Induced gamma $(35-45 \mathrm{~Hz})$ oscillations in ASD showed significant differences from controls at all eight sites of recording in facial emotion discrimination condition $(p<.05)$.

Discussion and Conclusions. These results indicate that more effort is required for an individual with Autism to recognize emotion rather than gender from viewing a face. Abnormal processing of emotional stimuli may provide an explanation for some of the social and communicative deficits observed in Autism. The results of the study contribute to better understanding of possible neurobiological mechanisms resulting in abnormal processing of facial information and in deficient social communication and mentalizing abnormalities in autism spectrum disorders.

\section{References}

Sabbagh, M. A. (2004). Understanding orbitofrontal contributions to theory-of-mind reasoning: Implications for autism. Brain and Cognition, 55(1), 209-219. http://dx.doi.org/10.1016 /j.bandc.2003.04.002

\section{Effects of Repetitive TMS on Autonomic Activity in Children with Autism Spectrum Disorder \\ Estate Sokhadze', Yao Wang ${ }^{2}$, Emily Casanova', Manuel Casanova', and Guela Sokhadze ${ }^{2}$ \\ ${ }^{1}$ University of South Carolina School of Medicine, Greenville, South Carolina, USA \\ ${ }^{2}$ University of Louisville, Louisville, Kentucky, USA}

Background. Autism is a pervasive developmental disorder marked by difficulty in social interaction, impairments or lack of communication, and restricted range of interests. In addition, many children with Autism exhibit symptoms associated with autonomic dysfunctions, which are presented as abnormalities in regulation of blood pressure, temperature, heart rate, and other body functions by the autonomic nervous system (ANS). The main findings of 
autonomic abnormalities studies in autism spectrum disorders (ASD) point at reduced baseline parasympathetic activity in association with evidence of increased baseline sympathetic tone resulting in autonomic disbalance, which negatively affects physiological functions and manifests in alterations of various electrophysiological measures.

Objectives. Aim of the study was to investigate electrophysiological measures reflecting ANS activity in two cohorts of children with ASD during 12 and 18 weekly sessions of the low-frequency repetitive Transcranial Magnetic Stimulation (rTMS) over dorsolateral prefrontal cortex (DLPFC). Hypotheses to be tested in the study were as follows: (1) lowfrequency $(0.5 \mathrm{~Hz})$ rTMS of prefrontal cortex may lower ANS hyperactivation in children with Autism through activation of frontal inhibitory tone controlling ANS, and (2) lower ANS arousal postTMS will be reflected in decrease of skin conductance level (SCL), heart rate (HR) and increased HR variability, and in improvement of behavioral evaluation scores.

Methods. We investigated autonomic activity in 30 children with ASD during 12 sessions of rTMS and 18 children with ASD during 18 sessions of rTMS over DLPFC. Physiological activity measures such as skin conductance level (SCL), heart rate (HR), and $H R$ variability (HRV) were recorded during rTMS sessions with a C-2 J\&J Engineering Inc. physiological monitoring system. Behavioral evaluations were conducted using Aberrant Behavior Checklist (ABC, Aman \& Singh, 1994) and Repetitive Behavior Scale (RBS-R, Bodfish, Symons, \& Lewis, 1999).

Results. Post 12-session rTMS measurements showed a decrease of low-frequency (LF) component of HRV with statistical changes in HR regression and standard deviation of HR, though without any significant changes in SCL. Post 18session rTMS outcomes showed slower heart rate accompanied by increase of high-frequency (HF) component of HRV (as indicated by R-R intervals of $E C G$ ), higher deviation of $R-R$, and lower $L F / H F$ ratio.

Conclusions. Our findings show reduced sympathetic activation after rTMS resulting in lower HR predominantly through withdrawal of sympathetic tone (LF of HRV) and increase of parasympathetic cardiac neural control activity post 12 rTMS sessions. Neuromodulation using prolonged 18session rTMS course in children with ASD resulted in a more pronounced HR slowing, a higher power of
HF of HRV and time domain measures of HRV after 18 rTMS course in Autism. Behavioral evaluations based on $A B C$ and RBS-R scores showed similar improvements in 12 and 18 sessions of rTMS. Lowfrequency rTMS activates inhibitory tone of the frontal cortex resulting in a lower excitation of the ANS probably through the inhibitory fronto-limbic circuits.

\section{References}

Aman, M. G., \& Singh, N. N. (1994). Aberrant Behavior Checklist - Community (Supplementary Manual). East Aurora, NY: Slosson Educational Publications.

Bodfish, J. W., Symons, F. J., and Lewis, M. H. (1999). Repetitive Behavior Scale. Western Carolina Center Research Reports.

\section{QEEG-Guided Neurofeedback for Autism Spectrum Disorder (ASD): A Validating Case Study \\ Justin Leiter, Jeremy London, and Thomas Collura}

ADD Centre and Biofeedback Institute of Toronto, Mississauga, Brain Enrichment Center, Bedford, Ohio, USA

Autism spectrum disorder (ASD) consists of persistent deficits in social communication and restricted repetitive patterns of behavior, interests, and/or activities (American Psychiatric Association, 2013). Individuals with ASD can also experience neurocognitive disturbances in voluntary behavior, social interaction/facial recognition, as well as executive function, specifically in shifting attention (Courchesne et al., 1994; Mosconi et al., 2009; Ozonoff, Pennington, \& Rogers, 1991). Treatment for ASD comes in the form of off-label medications, various behavioral interventions usually in the form of Early Intensive Behavioral Interventions (EBIB), and specialized diets such as gluten-free and casein-free or GFCF (Elder, 2008; Howlin, Magiati, \& Charman, 2009). Further research into additional interventions is needed. Currently, the implications of neurofeedback as a treatment for ASD are being explored, and recent research is promising.

Neurofeedback is on the rise as it offers noninvasive treatment through simple operant conditioning. Some neurofeedback has been focused on the suppression of theta power to increase the mindbody connection and thus decrease deficits in executive function, as well as a focus on the mirror neuron system (MNS) to improve social interaction. (Kouijzer, de Moor, Gerritis, Buitelaar, \& van Schie, 2009; Pineda, Carrasco, Datko, Pillen, \& Schalles, 2014). Both studies showed significant improvement. This case study will focus on quantitative electroencephalogram (qEEG)-guided 
neurofeedback as it allows for the individualization of treatment. Through qEEG mapping, unique dysregulations can be synchronized with the individual's reported and collected symptomology. This harmonization allows for neurofeedback to directly target the implicated dysfunction. In recent research, it has been found that a reduction in cerebral hyperconnectivity through qEEG-guided neurofeedback can lead to symptom relief (Coben \& Padolsky, 2007). Executive function deficits have also been relieved though qEEG-guided neurofeedback in individuals with ASD (Kouijzer, de Moor, Gerrits, Congedo, \& van Schie, 2009).

This case study will compare its treatment progression with recent literature, as well as reporting qEEG-guided neurofeedback's effectiveness on relieving psychological and neurocognitive disparities. This case study may also provide treatment guidance for neurofeedback clinicians with clients presenting with ASD.

\section{References}

American Psychiatric Association. (2013). Diagnostic and Statistical Manual of Mental Disorders (5th ed.). Washington, DC: Author.

Coben, R., \& Padolsky, I. (2007). Assessment-guided neurofeedback for autistic spectrum disorder. Journal of Neurotherapy, 11(1), 5-23. http://dx.doi.org/10.1300 IJ184v11n01 02

Courchesne, E., Townsend, J., Akshoomoff, N. A., Saitoh, O., Yeung-Courchesne, R., Lincoln, A. J., ... Lau, L. (1994). Impairment in shifting attention in autistic and cerebellar patients. Behavioral Neuroscience, 108(5), 848-865.

Elder, J. H. (2008). The gluten-free, casein-free diet in autism: An overview with clinical implications. Nutrition in Clinical Practice, 23(6), 583-588. http://dx.doi.org/10.1177 10884533608326061

Howlin, P., Magiati, I., \& Charman, T. (2009). Systematic review of early intensive behavioral interventions for children with autism. American Journal on Intellectual and Developmental Disabilities, 114(1), 23-41. http://dx.doi.org/10.1352 /2009.114:23-41

Kouijzer, M. E. J., de Moor, J. M. H., Gerrits, B. J. L., Buitelaar, J. K., \& van Schie, H. T. (2009). Long-term effects of neurofeedback treatment in autism. Research in Autism Spectrum Disorders, 3(2), 496-501. http://dx.doi.org/10.1016 /j.rasd.2008.10.003

Kouijzer, M. E. J., de Moor, J. M. H., Gerrits, B. J. L., Congedo, M., \& van Schie, H. T. (2009). Neurofeedback improves executive functioning in children with autism spectrum disorders. Research in Autism Spectrum Disorders, 3(1), 145-162. http://dx.doi.org/10.1016/j.rasd.2008.05.001

Mosconi, M. W., Kay, M., D'Cruz, A.-M., Seidenfeld, A., Guter, S., Stanford, L. D., \& Sweeney, J. A. (2009). Impaired inhibitory control is associated with higher-order repetitive behaviors in autism spectrum disorders. Psychological Medicine, 39(9), 1559-1566. http://dx.doi.org/10.1017/S0033291708004984

Ozonoff, S., Pennington, B. F., \& Rogers, S. J. (1991). Executive function deficits in high-functioning autistic individuals: Relationship to theory of mind. Journal of Child Psychology and Psychiatry, 32(7), 1081-1105. http://dx.doi.org/10.1111 /j.1469-7610.1991.tb00351.x
Pineda, J. A., Carrasco, K., Datko, M., Pillen, S., \& Schalles, M (2014). Neurofeedback training produces normalization in behavioural and electrophysiological measures of highfunctioning autism. Philosophical Transactions of the Royal Society B: Biological Sciences, 369(1644), 20130183. http://dx.doi.org/10.1098/rstb.2013.0183

Pop-Jordanova, N., Zorcec, T., Demerdzieva, A., \& Gucev, Z. (2010). QEEG characteristics and spectrum weighted frequency for children diagnosed as autistic spectrum disorder. Nonlinear Biomedical Physics, 4(1), 4-10. http://dx.doi.org/10.1186/1753-4631-4-4

Change of Power Spectral Density and Coherence Following Acupuncture Treatment in Patients with Insomnia Go Eun Lee 1 , Hyung Won Kang ${ }^{2}$, Lakhyung Kim ${ }^{3}$, Jung-Hwa Lim ${ }^{4}$, Jong-Hyang Yoo ${ }^{5}$, Sujeong Mun ${ }^{5}$, and Young Man Wei ${ }^{6}$

${ }^{1}$ Korean Rehabilitation Center, Seoul, South Korea

${ }^{2}$ Sanbon Hospital of Wonkwang University, Gunpo-city, Gyeonggi-do, South Korea

${ }^{3}$ Woosuk University, Jeonju, Jeonbuk, South Korea

${ }^{4}$ Pusan National University School of Korean Medicine, Yangsan, Gyeongsangnam, Korea

${ }^{5}$ Mibyeong Research Center, Korea Institute of Oriental Medicine, Daejeon, South Korea

${ }^{6}$ Hyooclinic, Seoul, South Korea

Background. Insomnia is one of the common symptoms in primary medical care. Insomnia usually distracts the attention and worsens daytime dysfunction and the depressive or anxious symptom. Recent studies of acupuncture for isomnia reported that acupuncture groups showed significant improvements compared with control groups. While the neurophysiological mechanism of acupuncture hasn't been revealed for insomnia, a few studies measured the effect of acupuncture treatment using qEEG. Our objective was to investigate the characteristics of the quantitative Electroencephalography (qEEG) at pre- and postacupuncture treatment on patients with insomnia.

Methods. Participants who have some problems initiating or maintaining sleep, or nonrestorative sleep more than 3 days a week and ISI over 8 and below 21, were treated by acupuncture for 2 weeks ( 3 times a week, total 6 times). We assessed the effectiveness of acupuncture for insomnia by ISI (Insomnia Severity Index), PSQI (Pittsburgh Sleep Quality Index), BDI (Beck Depression Inventory-K), STAI (State-Trait Anxiety Inventory-K), EQ-5D (EuroQol-5 Dimension), general health state VAS (Visual analogue scale). We also measured and analyzed power spectral density (delta $1-4 \mathrm{~Hz}$, theta 4-8 Hz, alpha 8-12 Hz, beta 12-25 Hz) and coherence at baseline and the end of treatment 
(second week) on linked ear montage using the NeuroGuide software program.

Results. Thirty-two participants were enrolled; 2 participants dropped out because of personal reasons. During the study period, there were no adverse events. The RMANOVA model demonstrated that total score of ISI was significantly decreased between baseline and the end of treatment (second week) and baseline and 2 weeks after treatment (fourth week), but not the end of treatment (second week) and 2 weeks after treatment (fourth week). During eye-closed state, on 01 in theta, T5 in beta, and 01 in beta, absolute power (AP) significantly increased. Moreover, on the same site in the same band, deviation from midpoint (zero) of z-scored absolute power (ZAP) also decreased significantly $(p<.05)$. The total number of $z$-scored absolute power exceed range of $90 \%( \pm 1.65)$ or $95 \%( \pm 1.96)$ decreased after treatment during eye-closed state, but not significantly. Coherence in delta (FP2-T4, Fz-T5, F4-T4, F8-T4, T3-T5, C3-T5, C3-P3, Cz-T5, C4-T4, T4-P4, T4-T6, T5-P3, T5-Pz, T5-O1, T5-O2, P3-Pz, P3-O1, Pz-O1), theta (T4-T6, T5-P3, T5-O1, T6-O2) and alpha (FP1-T5, FP2-T5, FP2-P3, F7-T5, F7-O2, F3-T5, Fz-T5, F4-T5, T3-O1, T3-O2, C3-O1, Cz-T5, T5-P4, T5-T6, T5-O2, P3-Pz, P3-P4, P3-T6, Pz-P4, $\mathrm{P} 4-\mathrm{O} 1, \mathrm{~T} 6-\mathrm{O} 1, \mathrm{~T} 6-\mathrm{O} 2)$ significantly increased on temporofrontal, temporooccipital, temporocentral and temporoparietal regions. Coherence in beta (FP1-O2, FP2-O2, F7-T6, F7-O1, F7-O2) significantly increased between frontal and occipital region.

Conclusion. Acupuncture would be effective for insomnia affecting neurophysiological aspects. The deviation of ZAP from midpoint (zero) was decreased after acupuncture treatment, but not significantly, and coherence-based functional network in theta and alpha range was increased between temporal and other regions. However, our study had a limitation of small sample size and noncontrol group. Therefore, a larger sample size and controlled study are needed to confirm the effectiveness and neurophysiololgical changes of acupuncture for insomnia.

\section{Acknowledgement}

This research was supported by grants from the Korea Institute of Oriental Medicine (K16091) and the Bio and Medical Technology Development Program of the National Research Foundation (NRF) funded by the Ministry of Science; ICT and Future Planning (NRF-2014M3A9D7034351).

\section{References}

Buysse, D. J., Germain, A., Hall, M. L., Moul, D. E., Nofzinger, E. A., Begley, A., ... Kupfer, D. J. (2008). EEG spectral analysis in primary insomnia: NREM period effects and sex differences. Sleep, 31(12), 1673-1682.

Cao, H., Pan, X., Li, H., \& Liu, J. (2009). Acupuncture for treatment of insomnia: A systematic review of randomized controlled trials. The Journal of Alternative and Complementary Medicine, 15(11), 1171-1186. http://dx.doi.org/10.1089/acm.2009.0041

Cho, Y. W., Shin, W. C., Yun, C. H., Hong, S. B., Kim, J., \& Earley, C. J. (2009). Epidemiology of insomnia in Korean adults: Prevalence and associated factors. Journal of Clinical Neurology, 5(1), 20-23. http://dx.doi.org/10.3988 /jcn.2009.5.1.20

Cortoos, A., De Valck, E., Arns, M., Breteler, M. H. M., \& Cluydts R. (2010). An exploratory study on the effects of teleneurofeedback and tele-biofeedback on objective and subjective sleep in patients with primary insomnia. Applied Psychophysiology and Biofeedback, 35(2), 125-134. http://dx.doi.org/10.1007/s10484-009-9116-z

Ernst, E., Lee, M. S., \& Choi, T.-Y. (2011). Acupuncture for insomnia? An overview of systematic reviews. The European Journal of General Practice, 17(2), 116-123. http://dx.doi.org /10.3109/13814788.2011.568475

Hammer, B. U., Colbert, A. P., Brown, K. A., \& Ilioi, E. C. (2011). Neurofeedback for insomnia: A pilot study of Z-score SMR and individualized protocols. Applied Psychophysiology and Biofeedback 36(4), 251-264. http://dx.doi.org/10.1007 /s10484-011-9165-y

Hsu, S.-F., Chen, C.-Y., Ke, M.-D., Huang, C.-H., Sun, Y.-T., \& Lin, J.-G. (2011). Variations of brain activities of acupuncture to TE5 of left hand in normal subjects. The American Journal of Chinese Medicine, 39(04), 673-686. http://dx.doi.org /10.1142/S0192415X11009111

Huang, W., Kutner, N., \& Bliwise, D. L. (2009). A systematic review of the effects of acupuncture in treating insomnia. Sleep Medicine Reviews, 13(1), 73-104. http://dx.doi.org /10.1016/j.smrv.2008.04.002

Kim, M. S., Cho, Y. C., Moon, J. H., \& Pak, S. C. (2009). A characteristic estimation of bio-signals for electroacupuncture stimulations in human subjects. The American Journal of Chinese Medicine, 37(03), 505-517. http://dx.doi.org /10.1142/S0192415X09007016

Kim, M. S., Kim, H. D., Seo, H. D., Sawada, K., \& Ishida, M. (2008). The effect of acupuncture at PC-6 on the electroencephalogram and electrocardiogram. The American Journal of Chinese Medicine, 36(3), 481-491. http://dx.doi.org/10.1142/S0192415X08005928

Krystal, A. D., Edinger, J. D., Wohlgemuth, W. K., \& Marsh, G. R. (2002). NREM sleep EEG frequency spectral correlates of sleep complaints in primary insomnia subtypes. Sleep, 25(6), 630-640.

Lee, S., Cheong, Y.-S., Park, E.-W., Choi, E.-Y., Yoo, H.-K., Kang, K.-H., ... Lee, H. (2010). Prevalence of sleep disorder and associated factors in family practice. Korean Journal of Family Medicine 31(11), 837-844. http://dx.doi.org/10.4082 $/ \mathrm{kjfm} .2010 .31 .11 .837$

Marzano, C., Ferrara, M., Sforza, E., \& De Gennaro, L. (2008). Quantitative electroencephalogram (EEG) in insomnia: A new window on pathophysiological mechanisms. Current Pharmaceutical Design, 14(32), 3446-3455. http://dx.doi.org $/ 10.2174 / 138161208786549326$

Perlis, M. L., Smith, M. T., Andrews, P. J., Orff, H., \& Giles, D. E. (2001). Beta/Gamma EEG activity in patients with primary and secondary insomnia and good sleeper controls. Sleep, 24(1), 110-117. 
Sarris, J., \& Byrne, G. J. (2011). A systematic review of insomnia and complementary medicine. Sleep Medicine Reviews, 15(2), 99-106. http://dx.doi.org/10.1016/j.smrv.2010.04.001

Staner, L., Cornette, F., Maurice, D., Viardot, G., Le Bon, O., Haba, J., ... Macher, J.-P. (2003). Sleep microstructure around sleep onset differentiates major depressive insomnia from primary insomnia. Journal of Sleep Research, 12(4), 319-330. http://dx.doi.org/10.1046/j.0962-1105.2003.00370.x

Streitberger, K., Steppan, J., Maier, C., Hill, H., Backs, J., \& Plaschke, K. (2008). Effects of verum acupuncture compared to placebo acupuncture on quantitative EEG and heart rate variability in healthy volunteers. The Journal of Alternative and Complementary Medicine, 14(5), 505-513. http://dx.doi.org/10.1089/acm.2007.0552

Suh, S., Kim, H., Yang, H.-C., Cho, E. R., Lee, S. K., \& Shin, C. (2013). Longitudinal course of depression scores with and without insomnia in non-depressed individuals: a 6-year follow-up longitudinal study in a Korean cohort. Sleep, 36(3), 369-376. http://dx.doi.org/10.5665/sleep.2452

Tan, H. J., Lan, Y., Wu, F. S., Zhang, H. D., Wu, L., Wu, X., \& Liang, F. R. (2014). Auricular acupuncture for primary insomnia: A systematic review based on GRADE system. Zhongguo Zhen Jiu = Chinese Acupuncture and Moxibustion, 34(7), 726-730.

Taylor, D. J., Mallory, L. J., Lichstein K. L., Durrence, H. H., Riedel, B. W., \& Bush, A. J. (2007). Comorbidity of chronic insomnia with medical problems. Sleep, 30(2), 213-218.

Yeung, W.-F., Chung, K.-F., Leung, Y.-K., Zhang, S.-P., \& Law, A. C. K. (2009). Traditional needle acupuncture treatment for insomnia: A systematic review of randomized controlled trials. Sleep Medicine, 10(7), 694-704. http://dx.doi.org/10.1016 /j.sleep.2008.08.012

Yeung, W.-F., Chung, K.-F., Poon, M. M.-K., Ho, F. Y.-Y., Zhang, S.-P., Zhang, Z.-J., ... Wong, V. T. (2012). Acupressure, reflexology, and auricular acupressure for insomnia: A systematic review of randomized controlled trials. Sleep Medicine, 13(8), 971-984. http://dx.doi.org/10.1016 /j.sleep.2012.06.003

Zhao, K. (2013). Acupuncture for the treatment of insomnia. International Review of Neurobiology, 111, 217-234. http://dx.doi.org/10.1016/B978-0-12-411545-3.00011-0

\section{Effects of Prefrontal Neurofeedback on Perceived Emotional State and Cognitive Functioning in Adolescents with Drug Abuse History: A Pilot Study Estate M. Sokhadze1, Jonathan Cowan' ${ }^{2}$, Yao Wang ${ }^{3}$, Manuel Casanova ${ }^{1}$, Eva Lamina ${ }^{3}$, and Allan Tasman ${ }^{3}$ ${ }^{1}$ University of South Carolina School of Medicine, Greenville, South Carolina, USA \\ ${ }^{2}$ Neurotek LLC, Goshen, Kentucky, USA \\ ${ }^{3}$ University of Louisville, Louisville, Kentucky, USA}

Background. In addition to well-known cognitive impairments, there are disruptions in processing emotion in individuals with substance dependence and in those predisposed to drug abuse (Sokhadze, Stewart, \& Hollifield, 2007). Neurofeedback trainingbased intervention is one of the potentially efficacious nonpharmacological treatment options for substance use disorders (Sokhadze, Cannon, \& Trudeau, 2008; Horrell et al., 2010). There have been used several neurofeedback protocols (e.g., Peniston \& Kulkosky, 1989; Scott, Kaiser, Othmer, \&
Sideroff, 2005, etc.) that reported success in treating addictive behaviors. However, there are practically no studies on the use of neurofeedback in occasional drug users who have drug use history but did not yet develop substance dependence (Sokhadze, Stewart, Tasman, Daniels, \& Trudeau, 2011). We developed protocol that might be used to prevent drug abuse through self-regulation training aimed to enhance EEG measures of positive emotional states.

Methods. One of the aims of this pilot study was to determine the dynamics of self-reported perceived positive emotional state rating before, during, and after twelve 25-min long neurofeedback training course in two groups of subjects. One group of subjects had documented drug use history $(N=6)$, most of them referred from Louisville Adolescent Network for Substance Abuse Treatment (LANSAT); and another one was a group of drug-naïve subjects $(N=6$, recruited mostly from undergraduate students). Our hypothesis was that the prefrontal gamma power increase over 12 training sessions is possible and will be accompanied by increased rating of positive affect. As a preferred neurofeedback protocol, we used enhancement of gamma range (centered around $40 \mathrm{~Hz}$ ) activity and inhibition/suppression of other frequencies at the prefrontal site (FPz).

Results and Discussion. Neurofeedback training at the midline prefrontal site after 12 sessions resulted as predicted in better performance on MicroCog and IVA+Plus tasks and improved scores on emotional self-reports (i.e., happiness) and clinical (BDI-II) status. Individual reports of selfreceived happiness scores assessed during each neurofeedback session using Continuous Response Digital Interface showed significant positive correlation with relative gamma power during individual training sessions. Analysis of EEG showed positive changes in the pattern of theta/beta ration and relative power of gamma. Neurofeedback was accompanied by positively correlated subjective self-reports of positive emotional feelings during sessions and resulted in improved performance on IVA+ and MicroCog tests during postneurofeedback evaluations. Posttraining evaluations and 3-month follow-up showed decrease in depression scores and increased happiness rating in both groups of subjects in this study.

Conclusions. Neurofeedback training aimed at inhibiting high amplitude EEG rhythms and upregulating high frequency rhythms in a group of adolescents at risk of drug abuse was accompanied 
by increase of self-reported rating of positive emotional states and completion of the course resulted in improved mood, enhanced performance on selective attention test and neurocognitive tests. The findings in this pilot warrant further research to investigate potential clinical efficacy of the method.

\section{References}

Horrell, T., El-Baz, A., Baruth, J., Tasman, A., Sokhadze, G., Stewart, C., \& Sokhadze, E. (2010). Neurofeedback effects on evoked and induced EEG gamma band reactivity to drugrelated cues in cocaine addiction. Journal of Neurotherapy, 14(3), 195-216. http://dx.doi.org/10.1080 /10874208.2010.501498

Peniston, E. G., \& Kulkosky, P. J. (1989). Alpha-theta brainwave training and beta-endorphin levels in alcoholics. Alcoholism: Clinical and Experimental Research, 13(2), 271-279. http://dx.doi.org/10.1111/j.1530-0277.1989.tb00325.x

Scott, W. C., Kaiser, D., Othmer, S., \& Sideroff, S. I. (2005). Effects of an EEG biofeedback protocol on a mixed substance abusing population. American Journal of Drug and Alcohol Abuse, 31(3), 455-469. http://dx.doi.org/10.1081 IADA-200056807

Sokhadze, E., Stewart, C. M., Tasman, A., Daniels, R., \& Trudeau, D. (2011). Review of rationale for neurofeedback application in adolescent substance abusers with comorbid disruptive behavioral disorders. Journal of Neurotherapy, 15(3), 232-261. http://dx.doi.org/10.1080 /10874208.2011.595298

Sokhadze, T., M., Stewart, C. M., \& Hollifield, M. (2007). Integrating cognitive neuroscience research and cognitive behavioral treatment with neurofeedback therapy in drug addiction comorbid with posttraumatic stress disorder: A conceptual review. Journal of Neurotherapy, 11(2), 13-44. http://dx.doi.org/10.1300/J184v11n02_03

Sokhadze, T. M., Cannon, R. L., \& Trudeau, D. L. (2008). EEG biofeedback as a treatment for substance use disorders: Review, rating of efficacy, and recommendations for future research. Applied Psychophysiology and Biofeedback, 33(1), 1-28. http://dx.doi.org/10.1007/s10484-007-9047-5

\section{EEG Phenotypes in Obsessive-Compulsive Disorder (OCD) \\ Antonio Martins-Mourao ${ }^{1}$ and Stig Hollup ${ }^{2}$ \\ ${ }^{1}$ Open University/London Scientific Neurotherapy, London, United Kingdom \\ ${ }^{2}$ Norwegian University of Science and Technology, Trondheim, Norway}

OCD is a complex and disabling form of anxiety disorder affecting $2-3 \%$ of the general population. It is characterized by recurrent intrusive thoughts (obsessions) that typically cause distress and by repetitive behavioral acts (compulsions) that are performed to reduce severe anxiety levels. Over $40 \%$ of patients currently fail to respond to psychotherapy and/or medication treatments (Bandelow, Seidler-Brandler, Becker, Wedekind, \& Rüther, 2007; Pallanti et al., 2002), especially when physicians base their judgments on the DSM-IV diagnostic categories and remain blind to patient neurophysiological subtypes (or EEG-phenotypes) underpinning the illness. Although knowledge of such subtypes has been found to predict depressed patients' response to medication (Cook et al., 2013), little is still known about the EEG-phenotypes in OCD.

The present study explores the relationship between EEG frequency bands and clinical symptoms of OCD. The EEG recordings of 60 patients with OCD and 60 healthy controls were correlated with behavioral measurements using the Yale Brown Obsessive Compulsive Scale (Y-BOCS) and the Minnesota Multiphasic Personality Inventory-2-RF (MMPI-2-RF). Statistical analyses revealed three main EEG phenotypes for OCD characterized by increased midline theta $(p<.01)$, frontal beta2 power $(p<.01)$, and a posterior beta1 power $(p<$ .01 ). A subgroup was also found to have increased alpha peak frequencies (i.e. $11 \mathrm{~Hz}$ or above), suggesting sympathetic overarousal. Pearson statistics showed that beta2 power correlated positively with obsession and compulsions severity (Y-BOCS) and thought dysfunction (MMPI-2-RF). Regression analysis showed significant correlation $(p<.05)$ between beta2 in posterior regions and total Y-BOCS score and several MMPI clinical scales. Applying a two-parameter linear model (obsession and compulsion score) suggested that this dependence is driven by the obsession component.

The above results suggest that the same DSMbased diagnostic category (i.e. OCD) may present very diverse neurophysiological profiles, or EEGphenoptypes. The use of the above data will predict appropriate medication for individual cases and is likely to increase the specificity and efficacy of future neurofeedback protocols for this patient group potentially leading to increased health comes.

\section{References}

Bandelow, B., Seidler-Brandler, U., Becker, A., Wedekind, D., \& Rüther, E. (2007). Meta-analysis of randomized controlled comparisons of psychopharmacological and psychological treatments for anxiety disorders. World Journal of Biological Psychiatry, 8(3), 175-187. http://dx.doi.org/10.1080 /15622970601110273

Cook, I. A., Hunter, A. M., Gilmer, W. S., losifescu, D. V., Zisook, S., Burgoyne, K. S., ... Leuchter, A. F. (2013). Quantitative electroencephalogram biomarkers for predicting likelihood and speed of achieving sustained remission in major depression: A report from the biomarkers for rapid identification of treatment effectiveness in major depression (BRITE-MD) trial. The Journal of Clinical Psychiatry, 74(1), 51-56. http://dx.doi.org/10.4088/JCP.10m06813

Pallanti, S., Hollander, E., Bienstock, C., Koran, L., Leckman, J., Marazziti, D., ... Zohar, J. (2002). Treatment non-response in 
OCD: Methodological issues and operational definitions. International Journal of Neuropychopharmacology, 5(2), 181191. http://dx.doi.org/10.1017/S1461145702002900

Equivalence of a Continuous EEG Discrimination Task to Standard Operant Control Training Jon Frederick ${ }^{1}$, Casey Klein ${ }^{2}$, Heim Andrew ${ }^{2}$, Kelli Dunn $^{3}$, and Julia Schreiber ${ }^{2}$

${ }^{1}$ St. Cloud State University, Murfreesboro, Tennessee, USA

${ }^{2}$ Middle Tennessee State University, Murfreesboro, Tennessee, USA

${ }^{3}$ Centerstone, Nashville, Tennessee, USA

Biofeedback is commonly believed to train increased awareness and voluntary control over physiological processes that would otherwise remain unconscious and involuntary (Frederick, in press; Olson, 1987; Plotkin, 1981). Brener (1974) argued that repeated pairing of external feedback with internal afferents related to the response lead to the awareness and learning of a "response image," which allowed control of the response without external feedback. However, relatively little research has examined the relationship between awareness and control of physiological states. Most clinical and experimental biofeedback research has emphasized operant control, by rewarding subjects for producing desired physiological states. Discrimination training is another form of operant conditioning, that rewards subjects for increased awareness, that is, for correctly reporting their physiological state. For instance, the first historical report of operant conditioning of the EEG was a discrimination task in which subjects were prompted to guess whether they were in a high or low alpha amplitude state and immediately informed if their response was correct (Kamiya, 2011). Since similar kinds of selfmonitoring and evaluation have been found to facilitate learning of motor skills (Boutin, Blandin, Massen, Heuer, \& Badets, 2014; Kolovelonis, Goudas, \& Dermitzaki, 2011), we hypothesized that combining discrimination training with standard neurofeedback would facilitate learning of operant control of EEG alpha (8-12 Hz) amplitude. However, Heim, Dunn, Klein, Powers, and Frederick (2016) argued that Kamiya's "discrete" discrimination paradigm resulted in very slow learning because it provides relatively few trials per minute. The present study examined the effect of a continuous discrimination task (CDT) on learned operant control of EEG alpha. In the CDT, participants manipulated a controller indicating their subjective rating of their alpha amplitude on a 10-point scale. The changing pitch of a tone (presented about 120/min) represented the absolute difference between the participant's rating and their actual alpha amplitude. One group $(n=9)$ received seven 40-min sessions of standard operant control training to increase and decrease alpha in eight alternating 5-min runs. The CDT-mixed group $(n=9)$ received only half of that training per session, instead performing the CDT during the 11-20th and 31-40th minutes of each session. Performance in the alpha operant control task was defined as the percent average difference between the increase and decrease conditions. The CDT group performed nonsignificantly higher than the control group (mean session improvement over first session baseline $14.0 \%$ vs. $6.8 \%$, one-tailed $t[16]=0.822, p=.22, d=0.39$ ). Although these results do not demonstrate that the CDT facilitates learning of voluntary control of EEG alpha, the fact that the CDT can be substituted for standard operant control training during half of the session time without a deficit in learning suggests that these two tasks are functionally equivalent and may have substantial similarity in the skills required. While results in this sample of mostly normal subjects show a small effect size, the CDT may have some clinical utility to provide additional motivation to clients who show deficient attention toward standard neurofeedback tasks.

\section{References}

Boutin, A., Blandin, Y., Massen, C., Heuer, H., \& Badets, A. (2014). Conscious awareness of action potentiates sensorimotor learning. Cognition, 133(1), 1-9. http://dx.doi.org/10.1016/j.cognition.2014.05.012

Brener, J. (1974). A general model of voluntary control applied to the phenomena of learned cardiovascular change. In Obrist, P., Black, A. H., Brener, J., \& DiCara, L. V. (Eds.), Cardiovascular psychophysiology. Chicago: Aldine.

Frederick, J. A. (2012). Psychophysics of EEG alpha state discrimination. Consciousness and Cognition, 21(3), 13451354. http://dx.doi.org/10.1016/j.concog.2012.06.009

Frederick, J. A. (in press). EEG state discrimination and the phenomenal correlates of brainwave states. In T. F. Collura \& J. A. Frederick (Eds.), Handbook of Clinical QEEG and Neurotherapy (annotated and with an introduction by $\mathrm{J}$. Kiffer). New York: Taylor \& Francis.

Heim, A. S., Dunn, K. N., Klein, C. J., Powers, C. D., \& Frederick, J. A. (2016). Generalization of skills between discrimination and control of EEG alpha. Presented at Association for Applied Psychophysiology and Biofeedback, Seattle, WA, March 2016.

Kamiya, J. (2011). The first communications about operant conditioning of the EEG. Journal of Neurotherapy, 15(1), 6573. http://dx.doi.org/10.1080/10874208.2011.545764

Kolovelonis, A., Goudas, M., \& Dermitzaki, I. (2011). The effect of different goals and self-recording on self-regulation of learning a motor skill in a physical education setting. Learning and Instruction, 21(3), 355-364. http://dx.doi.org/10.1016 /j.learninstruc.2010.04.001

Olson, P. (1987). Definitions of biofeedback. In Schwartz, M. S. (Ed.), Biofeedback: A practitioner's guide (pp. 33-38). New York: The Guilford Press.

Plotkin, W. B. (1981). A rapprochement of the operantconditioning and awareness views of biofeedback training: 
The role of discrimination in voluntary control. Journal of Experimental Psychology: General, 110(3), 415-428. http://dx.doi.org/10.1037/0096-3445.110.3.415
Received: November 18, 2016

Accepted: November 18, 2016

Published: December 8, 2016 